OPEN ACCESS

Edited by:

Lijun Bai,

Xi'an Jiaotong University, China

Reviewed by:

J. H. Gao,

Peking University, China Stefano Delli Pizzi, Università degli Studi G. d'Annunzio

Chieti e Pescara, Italy

*Correspondence:

Hui-Jie Li

lihj@psych.ac.cn

$\mathrm{Jie} L \mathrm{Lu}$

imaginglu@hotmail.com

Received: 29 April 2019 Accepted: 17 October 2019 Published: 06 November 2019

Citation:

Qi Z, An Y, Zhang M, Li H-J and Lu J (2019) Altered Cerebro-Cerebellar Limbic Network in AD Spectrum: A

Resting-State fMRI Study.

Front. Neural Circuits 13:72. doi: 10.3389/fncir.2019.00072

\section{Altered Cerebro-Cerebellar Limbic Network in AD Spectrum: A Resting-State fMRI Study}

\author{
Zhigang Qi ${ }^{1,2}$, Yanhong An ${ }^{1,2}$, Mo Zhang ${ }^{1,2}$, Hui-Jie $L i^{3 *}$ and Jie $L u^{1,2 *}$ \\ ${ }^{1}$ Department of Radiology, Xuanwu Hospital, Capital Medical University, Beijing, China, ${ }^{2}$ Beijing Key Laboratory of Magnetic \\ Resonance Imaging and Brain Informatics, Beijing, China, ${ }^{3}$ Key Laboratory of Behavioral Science, Institute of Psychology, \\ Chinese Academy of Sciences, Beijing, China
}

Recent evidence suggests that the cerebellum is related to motor and non-motor cognitive functions, and that several coupled cerebro-cerebellar networks exist, including links with the limbic network. Since several limbic structures are affected by Alzheimer pathology, even in the preclinical stages of Alzheimer's disease (AD), we aimed to investigate the cerebral limbic network activity from the perspective of the cerebellum. Twenty patients with mild cognitive impairment $(\mathrm{MCl}), 18$ patients with $A D$, and 26 healthy controls $(\mathrm{HC})$ were recruited to acquire Resting-state functional MRI (rs-fMRI). We used seed-based approach to construct the cerebro-cerebellar limbic network. Two-sample $t$-tests were carried out to explore the differences of the cerebellar limbic network connectivity. The first result, a sub-scale network including the bilateral posterior part of the orbitofrontal cortex (POFC) extending to the anterior insular cortex (AIC) and left inferior parietal lobule (L-IPL), showed greater functional connectivity in $\mathrm{MCl}$ than in $\mathrm{HC}$ and less functional connectivity in $\mathrm{AD}$ than in $\mathrm{MCl}$. The location of this sub-scale network was in accordance with components of the ventral attention network. Second, there was decreased functional connectivity to the right mid-cingulate cortex (MCC) in the AD and $\mathrm{MCl}$ patient groups relative to the $\mathrm{HC}$ group. As the cerebellum is not compromised by Alzheimer pathology in the prodromal stage of $A D$, this pattern indicates that the sub-scale ventral attention network may play a pivotal role in functional compensation through the coupled cerebro-cerebellar limbic network in $\mathrm{MCl}$, and the cerebellum may be a key node in the modulation of social cognition.

Keywords: mild cognitive impairment, Alzheimer's disease, cerebro-cerebellar connectivity, limbic network, resting-state functional MRI, compensation

\section{INTRODUCTION}

The most prominent feature of Alzheimer's disease (AD) is the compromise of episodic memory, even in its prodromal stage that is referred to as mild cognitive impairment (MCI). It is natural that the pathogenesis of the impairment of memory became a focus on the research of AD. Although it is also involved in memory function, the limbic system has attracted little attention in AD-related research (Rolls, 2015). However, the common neuropsychiatric symptoms (NPS) of AD, including agitation and aggression, can result in potential harm that affects the patients and their caregivers and may become a much more serious burden on the family than the amnesia (Craig et al., 2005). For most psychiatric conditions, dysfunction of the limbic structures affects emotion regulation, 
social interaction, and other behaviors. Understanding the limbic network would be meaningful for deeply probing brain functions, such as memory, and for clarifying the pathogenesis of psychiatric disorders, such as depression (Bennett, 2011), bipolar disorder (Leow et al., 2013), and psychosocial stress (Pruessner et al., 2008). To date, very few reports have focused on the significance of the limbic network in the evaluation of the biological underpinnings of AD (Trzepacz et al., 2013).

A series of clinical studies exploring NPS in patients with $\mathrm{MCI}$ and $\mathrm{AD}$ indicated that greater agitation was correlated with decreased gray matter (GM) density in the left insula and bilateral anterior cingulate (Bruen et al., 2008) and the left inferior frontal, insular, and bilateral retrosplenial cortices (Hu et al., 2015). Similarly, Trzepacz et al. (2013) reported that agitation and aggression severity was positively correlated to frontolimbic atrophy. Limbic dysfunction mainly includes the entorhinal cortex extending to the parietal cortex (Khan et al., 2014) and inferior frontal areas. Several limbic structures are involved by tau pathology even in $\mathrm{AD}$ patients (SpiresJones and Hyman, 2014). With combined positron emission tomography (PET) and magnetic resonance imaging (MRI), severe reductions of metabolism were observed throughout a network of limbic structures, including the hippocampus, medial thalamus, and posterior cingulate cortex (PCC) in mild AD patients; the same pattern was seen in amnestic MCI to a lesser degree (Nestor et al., 2003).

Resting-state functional MRI (rs-fMRI) can be used to evaluate the functional connectivity and large-scale functional network such as the default mode network (DMN) and limbic network. A typical finding is impairment of DMN activity, which is considered to be the origin of episodic memory damage in MCI and AD (Greicius et al., 2004; Li et al., 2015). Rs-fMRI may be particularly useful in the early detection of pathological change, due to neural function alterations may precede neuronal atrophy. Since the structure of the limbic system is compromised in MCI and $\mathrm{AD}$, it is reasonable to hypothesize that functional changes of the limbic system occur in $\mathrm{AD}$, even in the prodromal stages. A resting-state magnetoencephalography study on patients with MCI due to AD showed that patients with phosphorylated tau pathology had decreased functional connectivity such as the PCC, orbitofrontal cortex (OFC), and paracentral lobule, which could affect the limbic structures (Canuet et al., 2015).

The cerebellum has long been considered as mainly being involved in motor function; recently, it was also found to contribute to some non-motor cognitive functions. In other words, in addition to sensorimotor function, cognition, emotion, and autonomic functions can also be localized to the cerebellum, just like with the cerebrum. The cognitive/limbic cerebellum is found to be located in the cerebellar posterior lobe, which has been connected to cerebral cortex association areas. Moreover, lesions in the cerebellar posterior lobe lead to the cerebellar cognitive affective syndrome (CCAS). New evidence regarding cerebellar organization and functional connections have been provided by rs-fMRI studies in humans that have found distributed cerebral networks that underlie movement, attention, and limbic valence, as well as frontoparietal and default systems concerned with multiple different functions map onto the cerebellum with topographic specificity (Habas et al., 2009; O’Reilly et al., 2010). Patients with focal infarcts to the hemipons, present disrupted functional coupling between the cerebrum and contralateral cerebellum (Lu et al., 2011). Buckner et al. (2011) proposed an rs-fMRI-based approach to comprehensively explore the organization of cerebro-cerebellar circuits in the human brain. Seven networks, including the limbic network, within the cerebellum were observed to connect to the associated cerebral networks.

Because cerebellum plays crucial roles in higher cortical functions through a cerebro-cerebellar circuit, and it is not compromised by AD-related pathology in the early stages (Braak and Braak, 1991), here, we wanted to evaluate the pattern of cerebral limbic network activity of the AD spectrum through the coupled cerebro-cerebellar network.

\section{MATERIALS AND METHODS}

\section{Participants Recruitment}

Twenty patients with MCI, 18 patients with $\mathrm{AD}$, and 26 healthy controls (HC) were recruited in the study. The MCI and AD participants were recruited from the memory clinic of the Department of Neurology in hospital. HC were enrolled through volunteer posters from a community-based epidemiological study. Participants all provided written informed consent in accordance with the guidelines set by the Medical Research Ethics Committee of Beijing Xuanwu Hospital.

\section{Clinical Examination}

For each subject, clinical examination was composed of medical history, neurological examination, informant interview, and neuropsychological assessment including the Mini-Mental State Examination (MMSE) and Clinical Dementia Rating (CDR). Potential participants with a history of stroke, drug abuse, moderate to serious hypertension, psychiatric diseases, or other systemic diseases were excluded from the study.

$\mathrm{HC}$ did not have any subjective or reported cognitive impairments, and a CDR score of 0 and MMSE score $\geq 28$. The inclusion criteria for MCI patients were based on previous studies (Winblad et al., 2004; Zhang et al., 2017) and were as follows: had a subjective cognitive complaint (corroborated by an informant), episodic memory deficit on neuropsychological testing $(\mathrm{CDR}$ score $=0.5$ and MMSE score $>24$ ), and could complete daily living independently. AD patients met both the DSM-IV criteria for dementia and the National Institute of Neurological and Communicative Diseases and Stroke/AD and Related Disorders Association criteria for probable AD dementia.

The demographic and neuropsychological findings of the $\mathrm{AD}$, MCI, and HC groups are summarized in Table 1. Age, gender ratio, and years of education were matched across the three groups. The age of participants was similar between the three diagnostic groups (one-way ANOVA, $F=0.590, p=0.559$ ) with similar medians and ranges, whereas the MMSE scores were significantly different between the three groups (one-way ANOVA, $F=78.552, p<0.0001)$. 
TABLE 1 | Demographics of Alzheimer's disease (AD) and mild cognitive impairment (MCl) patients, and healthy controls (HC).

\begin{tabular}{|c|c|c|c|c|c|}
\hline & Age & Female/Male & Education (year) & MMSE & CDR \\
\hline AD patients $(n=20)$ & $73.1 \pm 6.7$ & $11 / 9$ & $10.5 \pm 0.6$ & $17.5 \pm 0.1$ & 1 \\
\hline $\mathrm{MCl}$ patients $(n=18)$ & $70.5 \pm 6.3$ & $10 / 8$ & $12.3 \pm 1$ & $26.1 \pm 0.6$ & 0.5 \\
\hline Healthy controls $(n=26)$ & $71.3 \pm 6.8$ & $12 / 14$ & $11 \pm 0.9$ & $28.3 \pm 0.5$ & 0 \\
\hline
\end{tabular}

No significant difference ( $p>0.05$ ) was observed in age, sex, or years of education between the three groups. Significant differences in Mini-Mental State Examination (MMSE) scores were seen between the three groups $(p<0.0001)$.

\section{MRI Data Acquisition}

All MRI data were acquired using a 3-T Siemens Trio system. Participants' heads were positioned within a 12-channel head coil. Foam padding was provided for comfort and to minimize head movement. During the rs-fMRI scanning, all participants were informed to close their eyes and to restrain from initiating attention-demanding activity. Functional images were collected using a gradient echo sequence [echo time $(\mathrm{TE})=40 \mathrm{~ms}$, repetition time $(\mathrm{TR})=2,000 \mathrm{~ms}$, flip angle $=90^{\circ}$, field of view $(\mathrm{FoV})=256 \mathrm{~mm}^{2}$, matrix $=64 \times 64$, 28 slices, slice thickness $=4 \mathrm{~mm}$, and $0 \mathrm{~mm}$ inter-slice gap] for a period of $8 \mathrm{~min}$ and $4 \mathrm{~s}$, resulting in a total of 239 imaging volumes. A T1-weighted anatomical image was also obtained using a magnetization-prepared rapid acquisition gradient echo sequence [TE $=2.2 \mathrm{~ms}$, $\mathrm{TR}=1,900 \mathrm{~ms}$, inversion time $(\mathrm{TI})=900 \mathrm{~ms}$, flip angle $=9^{\circ}$, FoV $=256 \mathrm{~mm}^{2}$, matrix $=224 \times 256,176$ slices, and $1 \mathrm{~mm}^{3}$ voxel]. 3D T1 structural images were collected for anatomical co-registration.

\section{MR Image Preprocessing and Individual Limbic Network Mapping}

We preprocessed all MRI data using the Connectome Computation System pipeline consisting of anatomical and functional image processing steps ( $\mathrm{Xu}$ et al., 2015). The anatomical preprocessing firstly denoised individual MRI structural images with a spatially adaptive non-local means filter (Xing et al., 2011). It stripped the skull of the denoised image and integrated manual edits to achieve a better brain extraction and segmented the brain volume into cerebrospinal fluid (CSF), white matter (WM), and GM tissues across both cerebral cortex and cerebellum. Individual pial (GM/CSF boundary) and white (GM/WM boundary) surfaces were subsequently generated and spatially normalized to match a group-level template surface in Montreal Neurological Institute (MNI) space. The subsequent functional preprocessing removed the first five volumes (10 s), detected and fixed temporal spikes by interpolation, corrected temporal acquisition difference in slice order and spatial head motion across volumes and performed intensity normalization on the $4 \mathrm{D}$ global mean intensity of 10,000 . This pipeline uses individual white surfaces to complete a boundary-based registration (BBR) for building spatial matching between multimodal (functional vs. anatomical) images in a single individual. Friston's 24-parameter motion curves, WM and CSF mean time series, as well as linear and quadratic trends were regressed out from the individual rs-fMRI time series using multiple linear regressions. As the final step in the CCS, the rs-fMRI time series were transferred into a $1-\mathrm{mm}$
MNI surface grid and down-sampled to the 4-mm MNI surface grid (fsaverage5).

Seed-based method was employed to construct a cerebrocerebellar limbic network. In this study, seed was chosen following these steps: first, cerebellar limbic network template was chosen from the 7-network parcellation of the cerebellum using 1,500 subjects (Buckner et al., 2011); second, the average time sequence of all the vertex in the template was extracted. Pearson's correlation coefficient between the individual mean time series of the cerebellar limbic network and preprocessed rs-fMRI time series of each vertex on the fsaverage5 was calculated and further converted into Fisher- $z$ value to quantify the limbic functional connectivity. This resulted in individual surface mapping of the limbic network connectivity for subsequent statistical tests across the three groups.

\section{Correlational Analysis}

Two-sample $t$-tests were applied to explore cerebellar limbic network connectivity differences between each pair of the three groups. Vertex-wise statistical cortical surface maps were corrected for multiple comparisons with cluster-level random field theory of family-wise errors (corrected $p<0.05$ ). Correlations between MMSE score and mean cerebellar limbic network connectivity across all vertices within each cluster exhibiting significant differences in functional connectivity were performed.

\section{RESULTS}

\section{Cerebral Areas Showing Significant Functional Connectivity to Cerebellar Limbic Network}

In $\mathrm{HC}$, a set of distributed areas showed positive functional connectivity to the cerebellar limbic network, including the frontal lobe, parietal lobe, and temporal lobe, mainly in the medial part of the hemispheres. Some areas also showed negative functional connectivity to the cerebellar limbic network, distributing in the convexity of the hemispheres (see Figure 1A). In the MCI group, fewer cerebral areas showed positive functional connectivity to the cerebellar limbic network than in $\mathrm{HC}$; however, the $t$-values were higher in some areas on visual inspection, mainly in the frontoparietal lobes (see Figure 1B). Many fewer areas exhibited positive functional connectivity to the cerebellar limbic network in AD than in MCI and HC; these areas were mostly distributed in the frontal and temporal pole (see Figure 1C). At the same time, the number of areas showing negative functional connectivity to the cerebellar limbic network was higher in MCI and AD groups than in the HC. These areas 


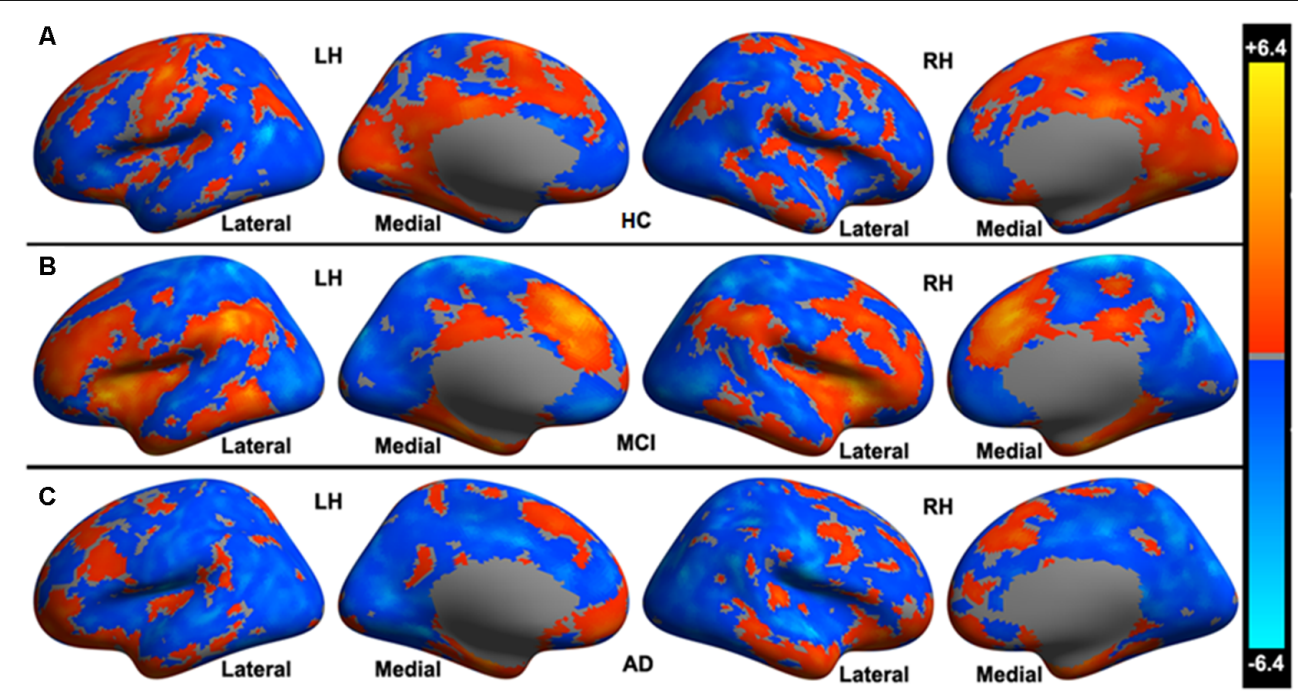

FIGURE 1 | Cerebral areas showing significant functional connectivity to the cerebellar limbic network in (A) healthy controls (HC), (B) mild cognitive impairment (MCl), and (C) Alzheimer's disease (AD) groups.

showing functional connectivity to the cerebellar limbic network were nearly symmetrically distributed in bilateral hemispheres.

\section{Significant Differences of the Functional Connectivity Between Cerebellar Limbic Network and Cerebral Cortex Between HC, $\mathrm{MCl}$, and $\mathrm{AD}$ Groups}

$\mathrm{HC}$ vs. $\mathrm{AD}$ : $\mathrm{HC}$ present increased functional connectivity in the right mid-cingulate cortex (MCC), right lingual gyrus to the cerebellar limbic network and decreased functional connectivity in the left temporal pole to cerebellar limbic network in comparison with AD (see Figure 2A).

$\mathrm{HC}$ vs. MCI: compared with MCI, HC show increased functional connectivity in the left paracentral lobule, right paracentral lobule extending to the right MCC to the cerebellar limbic network and decreased functional connectivity in the bilateral posterior part of the orbitofrontal cortex (POFC) extending to the anterior insular cortex (AIC), left inferior parietal lobule (L-IPL), and right fusiform gyrus to the cerebellar limbic network (see Figure 2B).

MCI vs. AD: increased functional connectivity between the bilateral POFC extending to the AIC (POFC-AIC), L-IPL and the cerebellar limbic network were found in MCI patients. No decreased functional connectivity was observed in MCI relative to $\mathrm{AD}$ (see Figure 2C).

Table 2 illustrated Talairach coordinates of clusters showing significant differences in functional connectivity with the cerebellar limbic network between HC, MCI, and AD groups.

\section{Relationships Between Functional Connectivity and MMSE Score}

A Pearson correlation was calculated between MMSE score and significant clusters. Among these clusters, the right
MCC (HC vs. AD), right lingual gyrus ( $\mathrm{HC}$ vs. $\mathrm{AD}$ ), bilateral posterior part of orbital-frontal cortex extending to the AIC (MCI vs. AD), and left inferior parietal lobule (MCI vs. AD) showed positive correlations with the MMSE score $(p<0.05)$. Negative correlation was observed between the left temporal pole (HC vs. AD) and MMSE score $(p<0.01$; see Table 3$)$. Clusters showing positive or negative correlation with the MMSE score are those with a significant difference in $\mathrm{HC}$ vs. $\mathrm{AD}$, and $\mathrm{MCI}$ vs. $\mathrm{AD}$. No significant correlations were observed between MMSE score and clusters showing significant differences between HC and MCI patients.

\section{DISCUSSION}

The current study evaluated changes in the cerebral limbic network in MCI and $\mathrm{AD}$ from the perspective of cerebrocerebellar functional connectivity because regions of the cerebellum are functionally coupled to specific cerebral networks (Buckner et al., 2011). In other words, we aimed to study the cerebral areas showing significant functional connectivity to the cerebellar limbic network and discriminate differences between $\mathrm{HC}, \mathrm{MCI}$, and $\mathrm{AD}$ groups. The pattern of the cerebral areas showing functional connectivity to the cerebellar limbic network, which was nearly symmetrically distributed in bilateral hemispheres, was similar in the three groups. The significant differences between the three group indicated that: (1) a sub-scale network, including the bilateral POFC-AIC and left IPL, showed greater functional connectivity in $\mathrm{MCI}$ relative to $\mathrm{HC}$ and lower connectivity in $\mathrm{AD}$ relative to MCI; (2) decreased functional connectivity in the left paracentral lobule and right paracentral lobule extending to right MCC in MCI relative to $\mathrm{HC}$ and decreased functional connectivity in the right $\mathrm{MCC}$ in $\mathrm{AD}$ relative to $\mathrm{HC}$ and 


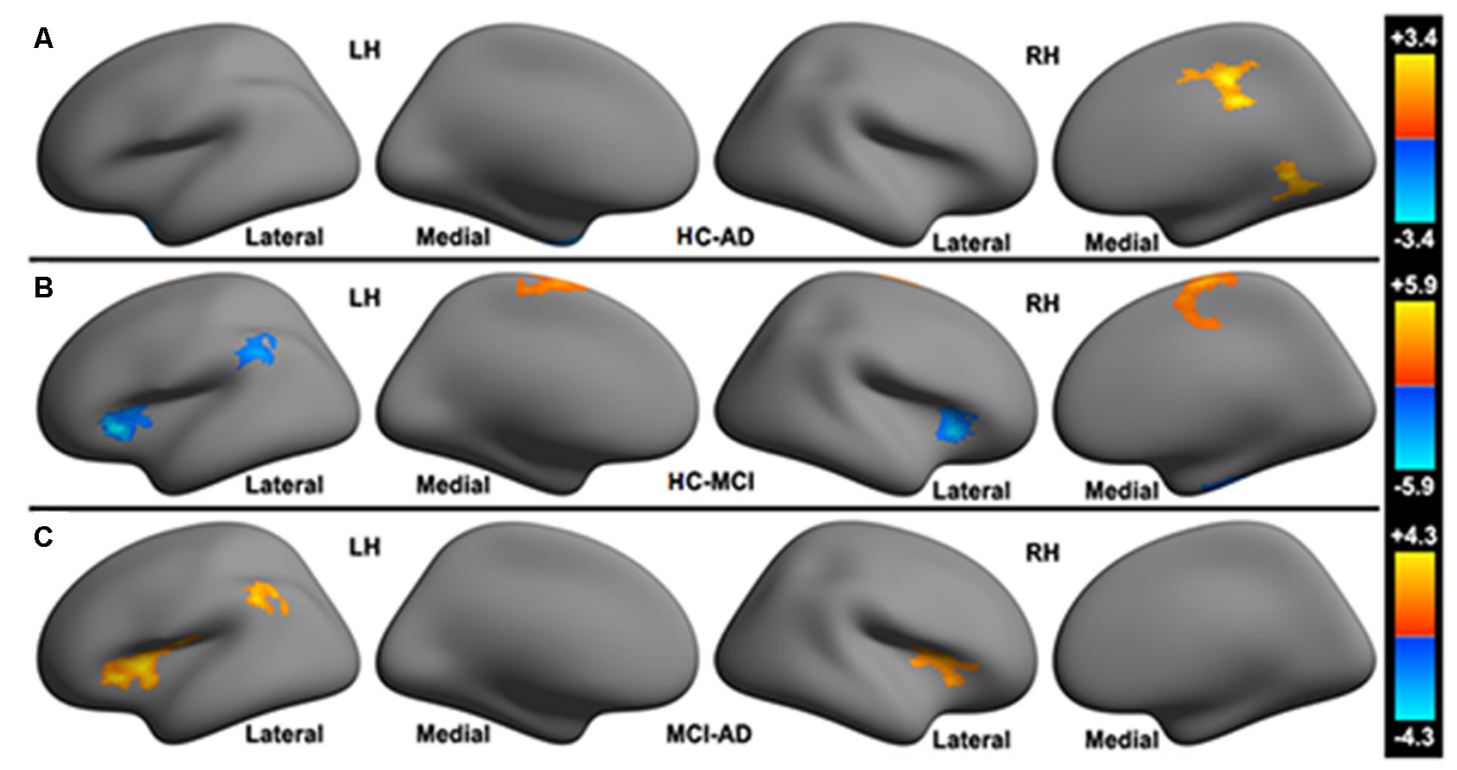

FIGURE 2 | Significant differences in the functional connectivity to the cerebellar limbic network between (A) $\mathrm{HC}$, (B) MCl and (C) AD groups.

TABLE 2 | Talairach coordinates of clusters showing significant differences in functional connectivity with the cerebellar limbic network between HC, MCI, and AD groups.

\begin{tabular}{|c|c|c|c|c|c|c|c|}
\hline & Size $\left(\mathrm{mm}^{2}\right)$ & $\mathbf{x}$ & $\mathbf{Y}$ & $\mathbf{Z}$ & BA & Area & $\mathbf{T}$ \\
\hline \multicolumn{8}{|l|}{$\mathrm{HC}-\mathrm{MCl}$} \\
\hline & 511 & -11 & -7 & 63 & 6 & Left paracentral lobule & 1.929 \\
\hline & 1,021 & 13 & -16 & 36 & 24 & Right mid-cingulate cortex/paracentral lobule & 3.376 \\
\hline & 639 & -55 & -47 & 30 & 40 & Left inferior parietal lobule & -1.915 \\
\hline & 559 & 39 & 22 & 8 & & Right posterior part of orbital-fronto cortex and anterior part of insular cortex & -2.554 \\
\hline & 499 & 39 & -11 & -23 & 20 & Right fusiform gyrus & -2.038 \\
\hline & 679 & -28 & 25 & 5 & & Left posterior part of orbital-fronto cortex and anterior part of insular cortex & -2.988 \\
\hline \multicolumn{8}{|l|}{$\mathrm{HC}-\mathrm{AD}$} \\
\hline & 624 & 13 & -16 & 36 & 24 & Right mid-cingulate cortex & 2.171 \\
\hline & 845 & 13 & -64 & 1 & & Right lingual gyrus & 1.851 \\
\hline & 539 & -31 & -6 & -28 & 20 & Left temporal pole & -2.022 \\
\hline \multicolumn{8}{|l|}{$\mathrm{MCl}-\mathrm{AD}$} \\
\hline & 1,314 & -47 & -12 & 17 & & Left posterior part of orbital-fronto cortex and anterior part of insular cortex & 5.043 \\
\hline & 703 & -49 & -45 & 43 & 40 & Left inferior parietal lobule & 2.192 \\
\hline & 734 & 32 & 14 & 10 & & Right posterior part of orbital-fronto cortex and anterior part of insular cortex & 2.865 \\
\hline
\end{tabular}

TABLE 3 | Significant correlations between the MMSE score and functional connectivity of brain regions.

\begin{tabular}{lcccccr}
\hline & R-MCC & R-LG & L-POFC-AIC & L-IPL & R-POFC-AIC & L-TP \\
\hline Correlation coefficient & 0.366 & 0.366 & 0.341 & 0.325 & 0.313 & -0.439 \\
$P$-value & 0.017 & 0.017 & 0.027 & 0.036 & 0.043 \\
\hline
\end{tabular}

Note: R-MCC, right mid-cingulate cortex; R-LG, right lingual gyrus; L-POFC-AIC, left posterior part of orbital-fronto cortex and anterior part of insular cortex; L-IPL, left inferior parietal lobule; R-POFC-AIC, right posterior part of orbital-fronto cortex and anterior part of insular cortex; L-TP, left temporal pole.

(3) decreased functional connectivity in the left temporal pole in $\mathrm{HC}$ relative to $\mathrm{AD}$ and in the right fusiform gyrus in $\mathrm{HC}$ relative to $\mathrm{MCI}$, increased functional connectivity in the right lingual gyrus in $\mathrm{HC}$ relative to $\mathrm{AD}$. The dual change of the cerebral limbic network in the current study is consistent with a previous study on MCI due to AD patients (Canuet et al., 2015). They found patients with abnormal CSF p-tau and A 342 levels showed both decreased and increased functional connectivity affecting limbic structures, with restingstate magnetoencephalography.

The limbic network was proposed by Papez (1995) to be a system involved in emotion and memory. It is well known that $\mathrm{AD}$ is characterized by impairment of episodic memory. However, since NPS was verified to occur in 
AD and MCI (Bruen et al., 2008; Trzepacz et al., 2013), and several previous studies have reported GM atrophy in the limbic network in AD and MCI (Bruen et al., 2008; Trzepacz et al., 2013; Hu et al., 2015), it is reasonable to consider that the limbic network is compromised in $\mathrm{AD}$ spectrum disorders.

Although the cerebellum has long been considered to be mainly involved in motor control, evidence has been accumulating that the cerebellum also contributes to higher cognitive function (Buckner, 2013; Bernard and Seidler, 2014; Sokolov et al., 2017; Schmahmann, 2019). Patients with left-sided lesions showed deficits in visuo-spatial processing while patients with right-sided lesions had verbal memory defects, indicating dysfunction of the contralateral cerebral hemispheres (Hokkanen et al., 2006). Other studies related to ADHD (Tomasi and Volkow, 2012), depression (Alalade et al., 2011), and aging (Bernard et al., 2013) without visible parenchymal lesions also revealed that cerebellar function might change accordingly. Moreover, a recent multi-study analysis showed that cerebellar activity during domain-specific mentalizing functionality is strongly connected with a corresponding mentalizing network in the cerebrum (Van Overwalle and Mariën, 2016). However, in the $\mathrm{AD}$ spectrum, the cerebellum was considered to be free from Alzheimer pathology until the severe phase of the disease, and always a normal reference in the evaluation of cerebral metabolism (Ni et al., 2013). Bai et al. (2011) reported that longitudinal functional connectivity between the hippocampus subregion and cerebellum may be a valuable biomarker to classify aMCI converters from aMCI nonconverters. Similarly, in a recent report (Delli Pizzi et al., 2019), hippocampal/entorhinal functional connectivity were evaluated in individuals with MCI those who did not convert to $\mathrm{AD}$ or show the presence of $\mathrm{AD}$ pathological load compared to those who converted to $\mathrm{AD}$ or showed presence of $\mathrm{AD}$ pathological load, increased functional connectivity to the selected regions, especially the cerebellum, were considered to be the strategy in maintaining the cognitive reserve. According to the MMSE and $\mathrm{CDR}$ score, $\mathrm{AD}$ patients in our study were classified as mild to moderate. It is feasible to illuminate the role of the cerebellum in the evaluation of the limbic network in the $\mathrm{AD}$ spectrum by examining coupled cerebro-cerebellar functional connectivity.

\section{POFC-AIC and IPL}

In the current study, the bilateral POFC-AIC, together with the left IPL, formed a sub-scale network that showed a dual pattern in $\mathrm{MCI}$ and $\mathrm{AD}$ patients. Increased functional connectivity between the sub-scale network and cerebellar limbic network was observed in MCI relative to HC and decreased functional connectivity was observed in $\mathrm{AD}$ relative to $\mathrm{MCI}$. The result provided support for a nonlinear trajectory of this sub-scale network activity in the evolution from MCI to $\mathrm{AD}$, similar to the pattern of memory-related neural activity reported by Celone et al. (2006). The OFC and other regions of the prefrontal cortex have been considered important in personality and social behavior. The lateral part of the OFC, which is also a component of limbic cortices, is mainly involved in behavioral inhibition, response inhibition, selective responses, and emotional cognitive adjustment. The OFC is not an area of onset for $\mathrm{AD}$ pathology in the brain; however, a large number of neurofibrillary tangles (NFTs) can be observed in the OFC in AD. The density of NFTs in the OFC was only secondary to that of the medial temporal lobe (MTL) even in healthy elderly with normal aging and MCI (Guillozet et al., 2003). These tangles may lead to the changes in OFC activity seen in $\mathrm{AD}$. The anterior insula is an important hub in the emotional limbic system, with the ventral part of anterior insula, just ventral to the primary taste cortex, a part of the limbic system. The ventral anterior insula, together with the anterior cingulate cortex (ACC) and MCC, form the visceromotor limbic cortices. Most researches on the insula have concentrated on its influence on affective disorders. In individuals with depression, task-induced fMRI study showed hypoactivation of the right insula in response to negative affective pictures (Lee et al., 2007), and resting-state fMRI showed decreased Regional Homogeneity ( $\mathrm{ReHo}$ ) in the right insula, which was positively correlated with anxiety severity (Yao et al., 2009). The ventral anterior insular region has strong projections to the $\mathrm{OFC}$ and receives inputs from the OFC and ACC (Price, 2007), involving in decoding and representing reward and punishment signals that produce autonomic/visceral responses.

The IPL is not a component of the limbic system and was considered to exhibit strong evidence of a direct role in episodic memory retrieval. Vilberg and Rugg (2008) speculated that the IPL is involved in the maintenance or representation of retrieved information in something like the episodic buffer. In a study of focal lateral parietal damage, Davidson et al. (2008) showed evidence of disrupted recollection in an anterograde memory task. Since some links from the emotional system to the memory system are present, we would like to speculate that the IPL might play a role in limbic activity from the connected network.

The bilateral POFC-AIC and left IPL showed a more positive correlation with the MMSE score in MCI than in AD $(p<0.05)$, whereas no significant difference in correlation was observed between HC and MCI. This indicates that this sub-scale network activity was correlated with Alzheimer pathology, and may be a key biomarker in limbic activity. Moreover, the difference between $\mathrm{HC}$ and MCI was much less than that between MCI and AD. Compensatory activity is thought to occur during the progression of $\mathrm{AD}$, especially in individuals at risk of $\mathrm{AD}$ (Bookheimer et al., 2000). That is to say, normal-appearing brain areas may be recruited for cognitive activity compensation in the progression of $\mathrm{AD}$ (Celone et al., 2006). In Yeo's Atlas (Yeo et al., 2011), the locations of bilateral POFC-AIC and left IPL are consistent with components of the ventral attention network, so we may speculate that limbic network activity compensation can be verified in MCI through a sub-scale ventral attention network, in addition to decompensation in $\mathrm{AD}$. This coincides with the progress of Alzheimer pathology.

\section{Paracentral Lobule and MCC}

The paracentral lobule and MCC showed a consistent change in $\mathrm{MCI}$ and $\mathrm{AD}$ relative to $\mathrm{HC}$. The left paracentral lobule 
and right paracentral lobule extending to right MCC showed compromised functional connectivity to the cerebellar limbic network in MCI, and right MCC in AD. Structural connectivity also showed shorter fibers in the cingulum connecting the MCC with adjacent areas, such as the paracentral lobule, and lingual and fusiform gyri. Generally, the sensorimotor function is spared until severe disease; therefore, the paracentral lobule was not considered to be involved in AD patients in the current study. However, recent reports showed some functional changes in this area, such as increased ReHo index in MCI (Wang et al., 2015), and a significant increase in the nodal centrality in APOE $\varepsilon 4$ carriers relative to APOE $\varepsilon 4$ non-carriers based on a graph theory brain network analysis (Yao et al., 2015). Unlike the results of previous reports, the paracentral lobule showed decreased functional connectivity in MCI in this study. The MCC is one part of the limbic cortex, belonging to the hippocampal-centric division, and is also part of the posterior part of the medial DMN. The Pearson correlation indicated that the right MCC showed a more positive correlation with the MMSE score in HC than in $\mathrm{AD}(p<0.05)$. No difference in correlation was observed between the right MCC and MMSE scores between HC and MCI, which is analogous to the pattern observed in the POFC-AIC and IPL.

\section{Fusiform and Lingual Gyri, and Temporal Lobe}

The fusiform gyrus is not a component of the cerebral limbic cortices, unlike the lingual gyrus. However, they both participate in mediating the perception of face identity (Hoffman and Haxby, 2000). The fusiform gyrus was also activated in a memory task. With associative encoding of novel picture-word pairs task, MCI subjects showed greater fMRI responses in the fusiform regions, which was believed to be a compensatory change due to the incipient atrophy in the anterior MTL (Hämäläinen et al., 2007). In the present study, the right fusiform showed increased functional connectivity in MCI relative to $\mathrm{HC}$, which was consistent with previous reports. Both the lingual gyrus and temporal pole are parts of the olfactocentric division of the limbic network (Catani et al., 2013). In the comparison between $\mathrm{AD}$ and $\mathrm{HC}$, the right lingual gyrus showed decreased while the temporal pole showed increased functional connectivity in AD. However, the correlation analysis showed that a positive correlation with MMSE score was observed in the right lingual gyrus and a negative correlation observed in the right temporal pole. To some degree, the negative correlation between the left temporal pole and MMSE score may indicate that the lower MMSE score in $\mathrm{AD}$ patients corresponds to stronger functional activity in this area. Mesulam (1998) has theorized that the temporal poles act as "transmodal epicenters" where information from the multiple sensory modalities is combined to form complex, symbolic, personalized representations. This area was always focused in the study of frontotemporal dementia, especially in the behavioral variant frontotemporal dementia (Hornberger et al., 2011). Zahn et al. (2009) showed that OFC and temporal pole atrophy was associated with disinhibited social behavior. The interaction of the OFC with temporal regions via the uncinate fasciculus might be crucial in maintaining normal behavior (Green et al., 2010). A large-scale functional connectivity study showed that the medial part of the left temporal pole is connected to paralimbic structures (Pascual et al., 2015). We should consider that the temporal pole is also a pivotal area in the evaluation of $\mathrm{AD}$ and deserves to be studied further.

\section{CONCLUSION}

The current study investigated the pattern of limbic network activity in the $\mathrm{AD}$ spectrum by examining the cerebro-cerebellar functional connectivity as the cerebellum is involved in the modulation of cognitive function. Functional connectivity to the cerebellar limbic network was significantly more compromised in $\mathrm{AD}$ than in MCI patients. This coincides with the progress of Alzheimer pathology. However, the dual pattern of the sub-scale ventral attention network, increased in MCI vs. HC and decreased in $\mathrm{AD}$ vs. $\mathrm{MCI}$, indicating a compensatory mechanism in the MCI period, may be significant in the illumination of the limbic network in the $\mathrm{AD}$ spectrum. And this is consistent with Delli's report (Delli Pizzi et al., 2019) that increased functional connectivity between the hippocampus and the cerebellar functional associated regions, including the limbic system, was observed in individuals with MCI those who did not convert to $\mathrm{AD}$ compared to those who converted to $\mathrm{AD}$. In Skouras' report (Skouras et al., 2019), cerebellum showed increased functional connectivity to PCC in asymptomatic preclinical $\mathrm{AD}$ and to $\mathrm{MCC}$ in MCI, these were also considered as functional compensation. Considering that the cerebellum was spared in the early stages of the $\mathrm{AD}$, it is reasonable to believe that the cerebellum is crucial in this process. In addition, the change in left temporal pole activity supports the notion that more attention should be paid to this area in the limbic network in AD.

\section{LIMITATION}

Although this is a pilot study, we think it was meaningful to investigate the cerebral limbic network activity from the perspective of coupled cerebro-cerebellar functional connectivity. Nonetheless, there were some limitations in this study. The first was the small sample in each group. The second item may be the incomplete neuropsychological study without the use of the Neuropsychiatric Inventory (NPI). Third, we believe it would be more helpful to take the DMN into consideration and analyze the interrelationship between them.

\section{DATA AVAILABILITY STATEMENT}

The raw data supporting the conclusions of this manuscript will be made available by the authors, without undue reservation, to any qualified researcher.

\section{ETHICS STATEMENT}

The study was approved by the local ethics committee of Xuanwu Hospital. Written informed consent was obtained from 
all participants in accordance with the Declaration of Helsinki prior to the study.

\section{AUTHOR CONTRIBUTIONS}

ZQ, H-JL and JL contributed to the conception and design of the study. ZQ organized the database. H-JL performed the statistical analysis. ZQ, YA and MZ wrote the first draft of the manuscript. All authors contributed to manuscript revision, read and approved the submitted version.

\section{REFERENCES}

Alalade, E., Denny, K., Potter, G., Steffens, D., and Wang, L. (2011). Altered cerebellar-cerebral functional connectivity in geriatric depression. PLoS One 6:e20035. doi: 10.1371/journal.pone.0020035

Bai, F., Xie, C., Watson, D. R., Shi, Y., Yuan, Y., Wang, Y., et al. (2011). Aberrant hippocampal subregion networks associated with the classifications of aMCI subjects: a longitudinal resting-state study. PLoS One 6:e29288. doi: 10.1371/journal.pone.0029288

Bennett, M. R. (2011). The prefrontal-limbic network in depression: Modulation by hypothalamus, basal ganglia and midbrain. Prog. Neurobiol. 93, 468-487. doi: 10.1016/j.pneurobio.2011.01.006

Bernard, J. A., Peltier, S. J., Wiggins, J. L., Jaeggi, S. M., Buschkuehl, M., Fling, B. W., et al. (2013). Disrupted cortico-cerebellar connectivity in older adults. Neuroimage 83, 103-119. doi: 10.1016/j.neuroimage.2013. 06.042

Bernard, J. A., and Seidler, R. D. (2014). Moving forward: age effects on the cerebellum underlie cognitive and motor declines. Neurosci. Biobehav. Rev. 42, 193-207. doi: 10.1016/j.neubiorev.2014.02.011

Bookheimer, S. Y., Strojwas, M. H., Cohen, M. S., Saunders, A. M., PericakVance, M. A., Mazziotta, J. C., et al. (2000). Patterns of brain activation in people at risk for Alzheimer's disease. N. Engl. J. Med. 343, 450-456. doi: 10.1056/NEJM200008173430701

Braak, H., and Braak, E. (1991). Neuropathological stageing of Alzheimer-related changes. Acta Neuropathol. 82, 239-259. doi: 10.1007/bf00308809

Bruen, P. D., McGeown, W. J., Shanks, M. F., and Venneria, A. (2008). Neuroanatomical correlates of neuropsychiatric symptoms in Alzheimer's disease. Brain 131, 2455-2463. doi: 10.1093/brain/awn151

Buckner, R. L. (2013). The cerebellum and cognitive function: 25 years of insight from anatomy and neuroimaging. Neuron $80,807-815$. doi: 10.1016/j.neuron. 2013.10.044

Buckner, R. L., Krienen, F. M., Castellanos, A., Diaz, J. C., and Yeo, B. T. (2011). The organization of the human cerebellum estimated by intrinsic functional connectivity. J. Neurophysiol. 106, 2322-2345. doi: 10.1152/jn.00339.2011

Canuet, L., Pusil, S., López, M. E., Bajo, R., Pineda-Pardo, J. Á., Cuesta, P., et al. (2015). Network disruption and cerebrospinal fluid amyloid-beta and phospho-tau levels in mild cognitive impairment. J. Neurosci. 35, 10325-10330. doi: 10.1523/jneurosci.0704-15.2015

Catani, M., Dell'acqua, F., and Thiebaut de Schotten, M. (2013). A revised limbic system model for memory, emotion and behaviour. Neurosci. Biobehav. Rev. 37, 1724-1737. doi: 10.1016/j.neubiorev.2013.07.001

Celone, K. A., Calhoun, V. D., Dickerson, B. C., Atri, A., Chua, E. F., Miller, S. L., et al. (2006). Alterations in memory networks in mild cognitive impairment and Alzheimer's disease: an independent component analysis. J. Neurosci. 26, 10222-10231. doi: 10.1523/JNEUROSCI.2250-06.2006

Craig, D., Mirakhur, A., Hart, D. J., McIlroy, S. P., and Passmore, A. P. (2005). A cross-sectional study of neuropsychiatric symptoms in 435 patients with Alzheimer's disease. Am. J. Geriatr. Psychiatry 13, 460-468. doi: 10.1176/appi. ajgp.13.6.460

Davidson, P. S., Anaki, D., Ciaramelli, E., Cohn, M., Kim, A. S., Murphy, K. J., et al. (2008). Does lateral parietal cortex support episodic memory? Evidence from focal lesion patients. Neuropsychologia 46, 1743-1755. doi: 10.1016/j. neuropsychologia.2008.01.011

\section{FUNDING}

This work was supported by Beijing Municipal Geriatric Medical Research Center Project (PXM2019_026283_000003), Beijing Municipal Commission of Science and Technology (Z171100000117001), Beijing Municipal Administration of Hospitals' Ascent Plan (DFL20180802), Natural Science Foundation of China (81141018, 31871143), and National Key Research and Development Program of China (2016YFC0107100).

Delli Pizzi, S., Punzi, M., Sensi, S. L., and Alzheimer's Disease Neuroimaging Initiative. (2019). Functional signature of conversion of patients with mild cognitive impairment. Neurobiol. Aging 74, 21-37. doi: 10.1016/j. neurobiolaging.2018.10.004

Green, S., Ralph, M. A., Moll, J., Stamatakis, E. A., Grafman, J., and Zahn, R. (2010). Selective functional integration between anterior temporal and distinct fronto-meso limbic regions during guilt and indignation. Neuroimage 52, 1720-1726. doi: 10.1016/j.neuroimage.2010.05.038

Greicius, M. D., Srivastava, G., Reiss, A. L., and Menon, V. (2004). Defaultmode network activity distinguishes Alzheimer's disease from healthy aging: evidence from functional MRI. Proc. Natl. Acad. Sci. U S A 101, 4637-4642. doi: 10.1073/pnas.0308627101

Guillozet, A. L., Weintraub, S., Mash, D. C., and Mesulam, M. M. (2003). Neurofibrillary tangles, amyloid, and memory in aging and mild cognitive impairment. Arch. Neurol. 60, 729-736. doi: 10.1001/archneur. 60.5 .729

Habas, C., Kamdar, N., Nguyen, D., Prater, K., Beckmann, C. F., Menon, V., et al. (2009). Distinct cerebellar contributions to intrinsic connectivity networks. J. Neurosci. 29, 8586-8594. doi: 10.1523/JNEUROSCI.1868-09.2009

Hämäläinen, A., Pihlajamäki, M., Tanila, H., Hänninen, T., Niskanen, E., Tervo, S., et al. (2007). Increased fMRI responses during encoding in mild cognitive impairment. Neurobiol. Aging 28, 1889-1903. doi: 10.1016/j.neurobiolaging. 2006.08.008

Hoffman, E. A., and Haxby, J. V. (2000). Distinct representations of eye gaze and identity in the distributed human neural system for face perception. Nat Neurosci. 3, 80-84. doi: 10.1038/71152

Hokkanen, L. S. K., Kauranen, V., Roine, R. O., Salonen, O., and Kotila, M. (2006). Subtle cognitive deficits after cerebellar infarcts. Eur. J. Neurol. 13, 161-170 doi: 10.1111/j.1468-1331.2006.01157.x

Hornberger, M., Geng, J., and Hodges, J. R. (2011). Convergent grey and white matter evidence of orbitofrontal cortex changes related to disinhibition in behavioural variant frontotemporal dementia. Brain 134, 2502-2512. doi: 10.1093/brain/awr173

$\mathrm{Hu}$, X., Meiberth, D., Newport, B., and Jessen, F. (2015). Anatomical correlates of the neuropsychiatric symptoms in Alzheimer's disease. Curr. Alzheimer Res 12, 266-277. doi: 10.2174/1567205012666150302154914

Khan, U. A., Liu, L., Provenzano, F. A., Berman, D. E., Profaci, C. P., Sloan, R., et al. (2014). Molecular drivers and cortical spread of lateral entorhinal cortex dysfunction in preclinical Alzheimer's disease. Nat. Neurosci. 17, 304-311. doi: $10.1038 / \mathrm{nn} .3606$

Lee, B. T., Cho, S. W., Khang, H. S., Lee, B. C., Choi, I. G., Lyoo, I. K., et al. (2007). The neural substrates of affective processing toward positive and negative affective pictures in patients with major depressive disorder. Prog. Neuropsychopharmacol. Biol. Psychiatry 31, 1487-1492. doi: 10.1016/j.pnpbp. 2007.06.030

Leow, A., Ajilore, O., Zhan, L., Arienzo, D., GadElkarim, J., Zhang, A., et al. (2013). Impaired inter-hemispheric integration in bipolar disorder revealed with brain network analyses. Biol. Psychiatry 73, 183-193. doi: 10.1016/j.biopsych.2012. 09.014

Li, H. J., Hou, X. H., Liu, H. H., Yue, C. L., He, Y., and Zuo, X. N. (2015). Toward systems neuroscience in mild cognitive impairment and Alzheimer's disease: a meta-analysis of 75 fMRI studies. Hum. Brain Mapp. 36, 1217-1232. doi: 10.1002/hbm.22689 
Lu, J., Liu, H., Zhang, M., Wang, D., Cao, Y., Ma, Q., et al. (2011). Focal pontine lesions provide evidence that intrinsic functional connectivity reflects polysynaptic anatomical pathways. J. Neurosci. 31, 15065-15071. doi: 10.1523/jneurosci.2364-11.2011

Mesulam, M. M. (1998). From sensation to cognition. Brain 121, 1013-1052. doi: 10.1093/brain/121.6.1013

Nestor, P. J., Fryer, T. D., Smielewski, P., and Hodges, J. R. (2003). Limbic hypometabolism in Alzheimer's disease and mild cognitive impairment. Ann. Neurol. 54, 343-351. doi: 10.1002/ana.10669

Ni, R., Gillberg, P. G., Bergfors, A., Marutle, A., and Nordberg, A. (2013). Amyloid tracers detect multiple binding sites in Alzheimer's disease brain tissue. Brain 136, 2217-2227. doi: 10.1093/brain/awt142

O’Reilly, J. X., Beckmann, C. F., Tomassini, V., Ramnani, N., and JohansenBerg, H. (2010). Distinct and overlapping functional zones in the cerebellum defined by resting state functional connectivity. Cereb. Cortex 20, 953-965. doi: 10.1093/cercor/bhp157

Papez, J. W. (1995). A proposed mechanism of emotion. J. Neuropsychiatry Clin. Neurosci. 7, 103-112. doi: 10.1176/jnp.7.1.103

Pascual, B., Masdeu, J. C., Hollenbeck, M., Makris, N., Insausti, R., Ding, S. L., et al. (2015). Large-scale brain networks of the human left temporal pole: a functional connectivity MRI study. Cereb. Cortex 25, 680-702. doi: 10.1093/cercor/bht260

Price, J. L. (2007). Definition of the orbital cortex in relation to specific connections with limbic and visceral structures and other cortical regions. Ann. N Y Acad. Sci. 1121, 54-71. doi: 10.1196/annals. 1401.008

Pruessner, J. C., Dedovic, K., Khalili-Mahani, N., Engert, V., Pruessner, M., Buss, C., et al. (2008). Deactivation of the limbic system during acute psychosocial stress: evidence from positron emission tomography and functional magnetic resonance imaging studies. Biol. Psychiatry 63, 234-240. doi: 10.1016/j.biopsych.2007.04.041

Rolls, E. T. (2015). Limbic systems for emotion and for memory, but no single limbic system. Cortex 62, 119-157. doi: 10.1016/j.cortex.2013.12.005

Schmahmann, J. D. (2019). The cerebellum and cognition. Neurosci. Lett. 688, 62-75. doi: 10.1016/j.neulet.2018.07.005

Skouras, S., Falcon, C., Tucholka, A., Rami, L., Sanchez-Valle, R., Lladó, A., et al. (2019). Mechanisms of functional compensation, delineated by eigenvector centrality mapping, across the pathophysiological continuum of Alzheimer's disease. Neuroimage Clin. 22:101777. doi: 10.1016/j.nicl.2019.101777

Sokolov, A. A., Miall, R. C., and Ivry, R. B. (2017). The cerebellum: adaptive prediction for movement and cognition. Trends Cogn. Sci. 21, 313-332. doi: 10.1016/j.tics.2017.02.005

Spires-Jones, T. L., and Hyman, B. T. (2014). The intersection of amyloid beta and tau at synapses in Alzheimer's disease. Neuron 82, 756-771. doi: 10.1016/j. neuron.2014.05.004

Tomasi, D., and Volkow, N. D. (2012). Abnormal functional connectivity in children with attention-deficit/hyperactivity disorder. Biol. Psychiatry 71, 443-450. doi: 10.1016/j.biopsych.2011.11.003

Trzepacz, P. T., Yu, P., Bhamidipati, P. K., Willis, B., Forrester, T., Tabas, L., et al. (2013). Frontolimbic atrophy is associated with agitation and aggression in mild cognitive impairment and Alzheimer's disease. Alzheimers Dement. 9, S95.e1-S104.e1. doi: 10.1016/j.jalz.2012.10.005
Van Overwalle, F., and Mariën, P. (2016). Functional connectivity between the cerebrum and cerebellum in social cognition: a multi-study analysis. Neuroimage 124, 248-255. doi: 10.1016/j.neuroimage.2015.09.001

Vilberg, K. L., and Rugg, M. D. (2008). Memory retrieval and the parietal cortex: review of evidence from a dual-process perspective. Neuropsychologia 46, 1787-1799. doi: 10.1016/j.neuropsychologia.2008.01.004

Wang, Y., Zhao, X., Xu, S., Yu, L., Wang, L., Song, M., et al. (2015). Using regional homogeneity to reveal altered spontaneous activity in patients with mild cognitive impairment. Biomed Res. Int. 2015:807093. doi: 10.1155/2015/807093

Winblad, B., Palmer, K., Kivipelto, M., Jelic, V., Fratiglioni, L., Wahlund, L. O., et al. (2004). Mild cognitive impairment-beyond controversies, towards a consensus: report of the international working group on mild cognitive impairment. J. Intern. Med. 256, 240-246. doi: 10.1111/j.1365-2796.2004. 01380.x

Xing, X. X., Zhou, Y. L., Adelstein, J. S., and Zuo, X. N. (2011). PDE-based spatial smoothing: a practical demonstration of impacts on MRI brain extraction, tissue segmentation and registration. Magn. Reson. Imaging 29, 731-738. doi: 10.1016/j.mri.2011.02.007

Xu, T., Yang, Z., Jiang, L., Xing, X.-X., and Zuo, X.-N. (2015). A Connectome Computation System for discovery science of brain. Sci. Bull. 60, 86-95. doi: 10.1007/s11434-014-0698-3

Yao, Z., Hu, B., Zheng, J., Zheng, W., Chen, X., Gao, X., et al. (2015). A FDG-PET study of metabolic networks in Apolipoprotein $\mathrm{E} \in 4$ allele carriers. PLoS One 10:e0132300. doi: 10.1371/journal.pone.0132300

Yao, Z., Wang, L., Lu, Q., Liu, H., and Teng, G. (2009). Regional homogeneity in depression and its relationship with separate depressive symptom clusters: a resting-state fMRI study. J. Affect. Disord. 115, 430-438. doi: 10.1016/j.jad. 2008.10.013

Yeo, B. T., Krienen, F. M., Sepulcre, J., Sabuncu, M. R., Lashkari, D., Hollinshead, M., et al. (2011). The organization of the human cerebral cortex estimated by intrinsic functional connectivity. J. Neurophysiol. 106, 1125-1165. doi: 10.1152/jn.00338.2011

Zahn, R., Moll, J., Iyengar, V., Huey, E. D., Tierney, M., Krueger, F., et al. (2009). Social conceptual impairments in frontotemporal lobar degeneration with right anterior temporal hypometabolism. Brain 132, 604-616. doi: 10.1093/brain/awn343

Zhang, Y. W., Zhao, Z. L., Qi, Z., Hu, Y., Wang, Y. S., Sheng, C., et al. (2017). Local-to-remote cortical connectivity in amnestic mild cognitive impairment. Neurobiol. Aging 56, 138-149. doi: 10.1016/j.neurobiolaging.2017.04.016

Conflict of Interest: The authors declare that the research was conducted in the absence of any commercial or financial relationships that could be construed as a potential conflict of interest.

Copyright (C) 2019 Qi, An, Zhang, Li and Lu. This is an open-access article distributed under the terms of the Creative Commons Attribution License (CC BY). The use, distribution or reproduction in other forums is permitted, provided the original author(s) and the copyright owner(s) are credited and that the original publication in this journal is cited, in accordance with accepted academic practice. No use, distribution or reproduction is permitted which does not comply with these terms. 